\title{
Sexually transmitted diseases in children: adolescents
}

\author{
Frances M Cowan, Adrian Mindel
}

\section{Introduction}

Sexually active adolescents are the group at greatest risk of acquiring sexually transmitted infections. ${ }^{12}$ The adverse sequelae that occur as a consequence of some of these infections are particularly devastating in the very young. Teenagers have their entire reproductive future at risk and jeopardise their fertility by infection. Chronic viral infections with long latent periods may exert their influence at a younger age in those infected while teenagers. It is imperative that screening, treatment and prevention of sexually transmitted diseases (STDs) in this age group are regarded as a priority.

The increased vulnerability of sexually active adolescents to infection with STDs is likely to be multifactorial. There is good evidence to suggest that while males and females are reaching physical maturity at progressively younger ages, ${ }^{3}$ psychological maturation has not been similarly advanced. This widening gap in physical and psychological maturation, coupled with the unplanned and experimental nature of early sexual encounters increases the risk of unwanted pregnancy and of acquiring sexually transmitted infections. Young people with poorly developed "future time perspectives" cannot conceive that today's romantic encounters could lead to tomorrow's demise. ${ }^{4}$ Studies which have examined safe sex practises among adolescents have found there to be a low rate of barrier contraceptive use and poor knowledge about acquisition and prevention of sexually transmitted infections. ${ }^{5}$ Early age of first sexual intercourse is associated both with an increased number of lifetime sexual partners and an increased risk of acquiring sexually transmitted diseases. ${ }^{1}$ The age at which individuals first have sexual intercourse appears to be reducing. The National Survey of Family Growth (NSFG) examined sexual behaviour among 15-44 year old women in the USA and showed that the proportion of girls who had sexual intercourse by the age of 16 years was $4.6 \%$ in 1970 rising to $25.6 \%$ in 1988. This increase was most marked between 1985 and 1988. In addition, those adolescents who had sex at an early age were excluding infection from the upper genital tract, both nonspecifically by producing mucus which excludes various pathogens and antigens and by specific protective immune mechanisms, including humoral immunity, locally produced phagocytic cells and possibly cell mediated immunity. ${ }^{8}$ The enhancement of these host defenses occurs when ovulation begins (usually 2-3 years after the menarche). Cyclical progesterone is produced, promoting the defence role of cervical mucus. In addition, the squamo-columnar junction of the cervix lies on the ectocervix until several years after menarche, exposing the mucus-secreting columnar epithelium to potential pathogens. It is probable that this columnar epithelium is more susceptible to infection by $N$ gonorrhoeae and $C$ trachomatis than squamous epithelium and possibly to infection by other sexually transmitted agents (including carcinogens) as well. ${ }^{2}$ Little is known of the immunology and defense factors of the female genital tract prior to menarche or to the pubertal male genital tract.

Where and who should treat adolescent STD

Ideally open access, well publicised, confidential adolescent medicine clinics run by a multidisciplinary team should be available. These could provide contraceptive advice, health education, STD screening, HIV counselling and testing, pregnancy testing, abortion counselling, advise on substance abuse, psychological support and primary health care, with all the services on offer tailored to meet the specific needs of adolescents. By having all the services together at one site, a patient attending regarding concerns about substance abuse for example, could be encouraged to have STD screening and to use the contraceptive services.

In the early $1980 \mathrm{~s}$, a programme to improve the health care delivered to high-risk adolescents across the USA was instituted and has recently been evaluated.9 Patients attending specially funded adolescent health clinics which aimed to provide medical, social and mental health services to adolescents were compared with adolescents attending primary health care facilities that did not have specific adolescent facilities. While both types of clinic attracted a similar proportion of high-risk adolescents to their services, the adolescent medicine clinics detected a wider range of medical and behavioural problems than the comparison clinics. This was attributed to their more diverse and specially trained staff. \footnotetext{
more likely to have many sexual partners. While the data for adolescent males are less complete, they suggest that for each age cohort a higher proportion of boys than girls are sexually active. ${ }^{6}$

In women, the stage of physical maturation, may also affect their susceptibility to infection. ${ }^{7}$ The cervix has an important role in London W1N 8AA, UK F M Cowa

Accepted for publication 5 October 1992

of Genitourinary Medicine, Universit
} 
At present such clinics do not exist in the UK. Adolescents at risk of STDs are seen in adult genitourinary medicine clinics. While genitourinary physicians are trained to deal with both medical and psychological problems that arise in adults with sexually transmitted infection and psychosexual problems, the adult medicine system may not be able to give the more extensive education and support required by adolescents. Patients have to attend several different clinics to get advice on all aspects of adolescent health prevention. Extending STD screening facilities into teenage contraception clinics may partially fill this gap in the short term. In addition, genitourinary medicine departments could make their clinics more "adolescent friendly", perhaps with dedicated staff and set clinic times for teenagers. Wherever adolescents with STDs are screened and treated, there should be adequate support available for them. Increasing the awareness of all health care professionals who treat teenagers of the importance of screening and treating sexually transmitted infections may increase the proportion of sexually active teenagers screened.

\section{Patient confidentiality}

Sexual activity is one of the adult behaviours adolescents adopt when trying to distance the child-parent relationship. It is a means of asserting their independence. Parents frequently react with disapproval to the onset of sexual relations. Therefore confidentiality regarding sexual behaviour, sexual orientation and STDs is particularly important. If teenagers suspect that their parents will be informed about any aspect of their sexual behaviour they will be reluctant to attend clinics for screening or treatment of STDs and suffer the consequences of delayed diagnosis. Sometimes it will be appropriate to encourage adolescents to confide in their parents and in some circumstances it may help for the clinician to see adolescent and parents together. However, bringing undue pressure to bear is likely to be counterproductive.

\section{Contact tracing}

Contact tracing of sexual partners is of fundamental importance in STD control. Tracing sexual partners decreases the likelihood of recurrent infection once sex is resumed following treatment. In addition, it reduces the time from infection to presentation at hospital in the contact, if he or she have been infected. As adolescents with symptomatic disease present later than adults, contact tracing may lead to earlier presentation and hence reduce the risk of serious sequelae.

Adolescents may be reluctant to tell their sexual partners that they are at risk of infection. However, patients should be counselled that the referral and treatment of sexual contacts is imperative to prevent further disease transmission. In addition, patients should be warned that if they continue to have a sexual relationship with their unscreened contact they may become reinfected. The consequences of repeated infection should be stressed.
Psychological effects of STDs in adolescents Research which looks at the psychological and psychosexual consequences of being infected with a sexually transmitted disease is very limited, particularly in adolescents. Despite the outwardly permissive nature of our society there is still much stigma attached to diseases which are acquired sexually. This leads to feelings of guilt and shame in those infected. When the acquisition of a sexually transmitted infection implies infidelity within a relationship, feelings of betrayal and mistrust may also occur. In addition, those infected may not feel able to confide in friends or parents, which increases their feelings of isolation. Their future sexual relationships may be tainted by the worry of acquiring another infection. For people with longstanding conditions such as genital herpes or hepatitis B carrier status, the worry of infecting others may cause particular problems.

It is important that patients are given adequate information about their condition, such as the modes of transmission, the likelihood of infecting others, what symptoms to expect and the longterm sequelae. Without this, the patient has to rely on hearsay which may be misleading, further heightening their anxiety. Written information available in clinics or through other means is often aimed at infected adults and as such may be confusing rather than helpful.

With adequate explanation and sympathetic treatment, many of the worries surrounding infection can be allayed: It is important to recognise those for whom explanation and sympathy alone is inadequate so that they can be referred for further support and counselling. Clearly the degree of distress and the extent of support required will vary not only from patient to patient but also between the types of infection. A patient with uncomplicated non gonococcal urethritis, is less likely to require support than someone with frequently recurring genital herpes, for example.

Patients may indicate their anxiety in different ways. Some present repeatedly for "check-ups" to the clinic despite minimal risk of further infection and want all the screening tests repeated at each visit "in order to make sure they are clear of infection". Others present regularly with new infections, having had unprotected sex with many sexual partners, perhaps indicating lack of self-esteem and self-worth. It is important to be aware that this pattern of attendance may indicate underlying psychosexual morbidity and support should be offered as appropriate.

Presentation, diagnosis and treatment of sexually transmitted infections

Adolescents presenting to a genitourinary medicine department, clearly perceive themselves to be at risk of an STD and the diagnosis and treatment of any infection should proceed along similar lines to those in adults. It is perhaps more difficult to diagnose such infections in adolescents presenting to 
doctors in other specialties. The most important aspect of diagnosing and treating these teenagers is to be aware that STDs may occur. All adolescents in contact with medical services should have an assessment made of the degree of their sexual experience. If they may have been at risk of infection or pregnancy, onward referral to a department of genitourinary medicine and/or a family planning clinic should be considered. As many sexually transmitted infections are asymptomatic, the presence of symptoms should not be used as the basis for referral.

Adolescence is a time of sexual experimentation and is the time when heterosexuals are most likely to experiment with homosexual behaviour. Many people have "same-sex" experience during their teens. In addition, some teenagers are homosexual. It is therefore wrong to assume that sexually active means "heterosexually active". Gay, male adolescents or male heterosexual adolescents who have had same-sex experience are at greater risk of sexually transmitted infections than their heterosexual counterparts, occurring at both the same and different sites.

In addition to their increased risk of STDs, it is thought that non-virginal adolescents are more likely to participate in other high risk activities than their virginal counterparts. Several studies conclude that sexual intercourse in adolescence is "an element of a syndrome of problem behaviours that include drug and substance abuse, minor delinquency and school difficulty"..$^{11}$ In one study, sexually experienced adolescent girls (age 12-16 years) were $6 \cdot 3$ times more likely to report having attempted suicide. ${ }^{10}$ Drug and substance abuse is particularly worrying as unsafe, unprotected sex is most likely to occur while under the influence of alcohol or other substances, further increasing the risk of unplanned pregnancy and STDs. ${ }^{12}$ It seems appropriate, therefore, for the physician to ask about other risk behaviours during the consultation. An assessment of the level of the patient's knowledge regarding STDs, HIV infection, risk of pregnancy, contraception and safer sex practises should be made and if necessary further health education can be delivered. The patient should be encouraged to ask questions regarding any sexual or developmental concerns that they have.

Specific sexually transmitted infections

There are numerous sexually transmitted infections. In most circumstances the clinical features, diagnosis, treatment and possible complications are similar in adults and adolescents. Those conditions in which adolescents fare or behave differently from adults will be discussed. These include $N$ gonorrhoeae, $C$ trachomatis, urethritis, pelvic inflammatory disease, herpes simplex, human papilloma virus, syphilis and HIV infection.

Neisseria gonorrhoea Between 1975 and 1989 the number of cases of infection with $N$ gonorrhoeae in the USA reported to the Center for Disease Control, Atlanta decreased from
1 million to 750,000 . Despite the decline in most age groups, the gonorrhoea rates in both teenage males and females increased during the 1980s. Black teenagers had ten times higher $N$ gonorrhoeae rates than whites in 1981 and by 1989 this had risen to 44 times higher in black male teenagers and 16 times higher in black female teenagers. ${ }^{2}$ Twenty five percent of all cases of gonorrhoea reported to the CDC now occur in 15-19 year olds. ${ }^{13}$

Attendances with gonorrhoea at genitourinary medicine clinics in the UK declined steadily over the last decade until 1989. This decline was greatest among persons over 25 years old. ${ }^{14}$ Between 1988 and 1991, approximately one third (29-33\%) of all female cases of post pubertal gonorrhoea occurred in those aged under 20 years, with an eighth (13-15\%) of all male cases occurring in this age group.

Although there has been only limited research which has specifically looked at gonorrhoea in adolescents, the prevalence of infection among adolescent STD clinic attenders appears to be similar $(20 \%)$ in various cities in the UK. ${ }^{15}$ Female adolescents in residential care are particularly at risk of infection. ${ }^{16}$ As with other sexually transmitted infections, gonorrhoea may be isolated from asymptomatic individuals ${ }^{17-21}$ making symptoms of infection an insensitive means for deciding who should and should not be screened. Asymptomatic carriage is particularly common in female adolescents.

Chlamydia trachomatis The prevalence of genital chlamydial infection is highest among sexually active adolescents and has been shown to be between $10 \%$ and $25 \%$ in females. ${ }^{22}$ Those infected are often asymptomatic. ${ }^{23}$ Chlamydial infection in adolescent males has been much less investigated. It is likely, however, that the high prevalence in females is mirrored in males. Ten per cent $(11 / 105)$ of sexually active male military recruits in Sweden aged 17 and 18 years had evidence of urethral chlamydia infection although only $1 / 11$ was symptomatic of infection. ${ }^{24} C$ trachomatis causes more lower genital tract infections among teenagers than does $N$ gonorrhoeae. ${ }^{25}$

Since 1988, age-specific information relating to clinic attendances with chlamydia infection have been included in the routine STD statistics in the UK. As with gonorrhoea, approximately one third (28-33\%) of all female cases and one eighth (12\%) of all male cases occur in those aged under 20 years. As symptoms are not a reliable predictor of infection, chlamydia screening should be performed in all sexually active adolescents regardless of their symptomatology. This applies particularly to pregnant adolescents, whether seeking abortion or intending to continue with the pregnancy, because of their risk of pelvic inflammatory disease and of neonatal infection.

Urethritis The presentation and microbiological cause of urethritis appears to be simi- 
lar in adult and adolescent males. However, one study has indicated that males under 20 years are particularly at risk of chlamydial non gonococcal urethritis. ${ }^{26}$

Pelvic inflammatory disease (PID) In the USA more than one million women are hospitalised with pelvic inflammatory disease each year. Sixteen to twenty per cent of these are adolescents, ${ }^{1}$ with the age specific rates for PID (adjusting for sexual activity) highest among teenagers. ${ }^{27}$ It has been calculated that a sexually active 15 year old girl has ten times the risk of acquiring PID than that of her 24 year old counterpart. ${ }^{28}$ In addition, studies which have compared PID in adolescents with PID in adults have shown that adolescents are significantly more likely to present for treatment at a later stage in their disease, that infection with $N$ gonorrhoeae occurs more commonly in adolescents than adults and that the adolescents are more likely to be nulliparous. ${ }^{29}$ If current trends in adolescent PID continue it has been predicted that $10 \%$ of women will be sterile and $3 \%$ will have had an ectopic pregnancy by the end of the century..$^{28}$

Pelvic inflammatory disease is one of the most serious outcomes of sexually transmitted infection, frequently leading to tubal damage with its increased risk of ectopic pregnancy, tubal infertility and chronic pelvic pain. The risk of infertility following PID is influenced by the number of episodes of PID, the severity of inflammation, the length of time between disease onset and antimicrobial therapy and the contraceptive method used. ${ }^{30}{ }^{31}$ As adolescents attend for treatment later in their illness, they may be more prone to the long term sequelae. The majority of adolescents are nulliparous and have more reproductive years at risk than older women, this has serious implications for their future fertility and family.

In a prospective study of 100 female adolescents hospitalised with PID, Chlamydia trachomatis was isolated from the endocervix in $44.7 \%$ and Neisseria gonorrhoeae in $36.4 \% .{ }^{32}$

Although PID in adults is often treated on an outpatient basis, it may be wise to admit adolescents for their therapy. The risk to future fertility is considerable and adolescent drug compliance rates are reported to be only

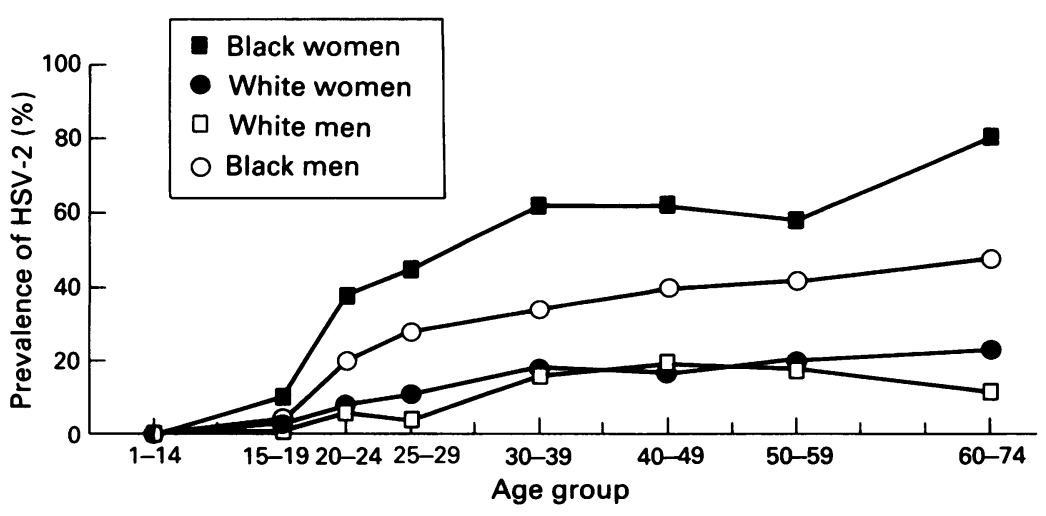

Prevalence of HSV-2 antibody in USA according to age, race and sex. (reprinted, by permission of the New England fournal of Medicine 1989;321:7-21)
$50 \% .{ }^{33}$ It is also easier to give psychosocial support while in hospital.

As in adults, one of the most important, but frequently neglected, aspects of treating PID is to trace contacts and treat the sexual partners.

Syphilis Until recently, the incidence of syphilis in the developed world was low, particularly among adolescents. In 1984, the rate of primary and secondary syphilis among 15-19 year olds in the US was only $17 / 100,000 .{ }^{13}$ In the UK, very few cases occur in teenages annually (17 cases in 1989 and 16 in 1990). For this reason screening sexually active adolescents for serological evidence of syphilis may be considered unnecessary. However, as the test is inexpensive and relatively easy to perform, screening may be worthwhile, particularly in certain populations. For example syphilis is relatively common among young male homosexuals. In addition rates of infection in any population can change over time. In the USA there has been a $34 \%$ increase in primary and secondary syphilis between 1981 and 1989.34 The populations affected have changed over time. The black to white incidence rate ratio was 14.5 in 1981 but had increased to 47.8 by 1989 . In Philadelphia there was a massive increase of $551 \%$ in the number of reported cases of early syphilis between 1985 and $1989 .{ }^{35}$ This epidemic, which is affecting many US states, is mainly among heterosexual males and females and appears to be related to crack use and prostitution..$^{36} \mathrm{~A}$ serious consequence of this epidemic is the alarming increase in the number of reported cases of congenital syphilis. There was an increase of more than $500 \%$ in the number of cases born in New York City between 1986 and $1988^{37}$ with $13 \%$ of these babies born to mothers under 20 years.

Herpes simplex virus Infections with herpes simplex virus type 1 and 2 (HSV 1 and 2) are amongst the commonest human viral infections. Many infected individuals are asymptomatic. Although both HSV 1 and 2 can be sexually transmitted, orolabial HSV infection due to HSV 1 commonly occurs in childhood following normal household contact. It is unusual for HSV 2 infection to occur prior to the onset of sexual activity (except very rarely as neonatal infection). A general population survey conducted between 1978-1980 in the USA suggested that the seroprevalence of HSV 2 increases with age. ${ }^{38}$ Antibodies to HSV 2 occurred rareiy under 15 years $(0.3 \%)$ but by 20 years between $5 \%$ (white males) and $28 \%$ (black females) had antibodies to HSV 2 (see fig). Other studies have shown that early first intercourse and many sexual partners are both associated with the presence of HSV 2 antibodies. ${ }^{39} 40$

Routine UK statistics (1988-1991) of clinic attendances for first attack of genital herpes indicate that $20 \%$ of all female attenders are under 20 years old, with $7 \%$ of male attendances below this age. 
Infection with herpes simplex is lifelong. The future course of the disease is impossible to predict. For this reason, many patients need psychological support. This may be particularly relevant in teenagers where the fear of having to tell all future sexual partners about their herpes infection may be overwhelming.

Human papilloma virus (HPV) infection and cervical dysplasia Genital HPV infection is the most commonly diagnosed viral STD in both the UK and the USA. ${ }^{41}$ It is usually sexually transmitted. In the absence of clinically apparent genital warts, evidence of HPV infection may be found on specimens taken for cervical cytology. Although cytological evidence of cervical HPV infection is most common in women between 20 and 24 years old one survey of 2,040 adolescent females aged 11-19 years attending a family planning clinic demonstrated an HPV prevalence of $5 \% .^{42}$

An increasing number of studies demonstrate an association between HPV infection (genotypes 16, 18 and 31) and cervical cancer. The relative frequency with which the various genotypes occur in adolescents has not been studied. However, the presence of HPV infection on cervical cytology does increase the likelihood of developing cervical cancer in the future. The age at which cervical cancer develops is decreasing; it is increasingly affecting young women..$^{43}$ It has been postulated that this is due to the changes in sexual behaviour which have occurred in the last 30 years.

The association of HPV infection and cervical dysplasia appears to be particularly marked in young women, with one study demonstrating a significant trend in the relative risk for in situ cervical cancer with age. Younger women showed a greater risk of carcinoma in situ following HPV infection on cervical cytology, with a relative risk of 38.7 among women aged less than 25 years when compared with the general population. ${ }^{44}$ This may be related to HPV infection of the biologically immature cervix. When adolescents with cervical intra-epithelial neoplasia were compared with adolescents attending a gynaecology clinic with symptoms of an STD, cervical biological immaturity appeared to be an important risk factor for the development of cervical intraepithelial neoplasia. ${ }^{45}$ If the association between HPV infection and cervical dysplasia is causal, the routine statistics for the UK which indicate that nearly $30 \%$ of all women attending GU medicine clinics with their first attack of genital warts are less than 20 years old, are particularly alarming.

$H I V$ infection It might seem reassuring that of the 218,301 AIDS patients in the USA by the end of March 1992, only $0.4 \%$ are teenagers; however, $19.5 \%$ are aged between 20-29 years. As the average incubation period of HIV infection is 8 years, it is likely that many of these young adults became infected as ado- lescents. ${ }^{46}$ A study of US military recruits demonstrated HIV seroprevalence of one in 3,000 for applicants aged 17-19 years old. ${ }^{47}$ Rates of infection among adolescent STD clinic attenders range from $0 \%$ to $4.6 \%$ (median $0.5 \%) .{ }^{48}$ When comparing the relative importance of various means of HIV transmission in the US it appears that the proportion of adolescents who became infected heterosexually is greater than for adults ( $9 \%$ vs $4 \%$ ). ${ }^{46}$ In addition, more HIV positive adolescents are female than male $(14 \%$ vs $7 \%$ ) and are black or hispanic rather than white $(17 \%$ vs $10 \%)$. Heterosexual infection of teenage girls has particularly serious implications as HIV infected women who become pregnant may infect their babies via vertical transmission. ${ }^{49}$ In New York $0.7 \%$ of teenage women delivering live births are HIV positive. ${ }^{50}$

Penetrative vaginal or anal sex is the sexual activity most likely to result in transmission of HIV infection..$^{51}$ While anal intercourse is the most efficient means of sexual transmission, vaginal intercourse is the most important route worldwide. In both cases the risk of transmission appears to be reduced by the conscientious use of condoms. ${ }^{52}$ Unfortunately very little is known about the relative frequency of these types of sexual behaviour in adolescence. One study based on an urban adolescent outpatient clinic in the USA found that $26 \%$ of teenagers had practised anal intercourse, but only two thirds of these used a condom..$^{53}$

If adolescents are having sex with other adolescents the low rate of teerlage HIV infection is reassuring. However, the sexual partners of adolescent male homosexuals who have intercourse are on average 7 years older..$^{54}$ In addition studies which have investigated sexual behaviour in adolescents in the context of HIV infection have shown that condom use is disappointingly low and that teenagers continue to take risks. ${ }^{55} 12$ Unsafe sexual behaviour is more likely to occur following alcohol consumption. ${ }^{12}$

There is no cure for HIV infection and the treatment currently available has only limited effect. The outlook for patients with AIDS is grim. It is unlikely that an effective vaccine will be available for some time. For these reasons, the only currently possible effective strategy with which to combat the HIV epidemic is one of prevention of infection.

It is imperative that all individuals are educated about the routes of HIV transmission and about safer sex and safe injecting techniques. An effective AIDS-prevention programme must reach all those at risk of infection and not just those who are easily accessible. Unfortunately the teenagers most at risk of HIV infection are likely to be the hardest to reach (school truants, drug users, runaways, etc.) and special efforts must be made to contact them. The information teenagers receive must be acceptable, clear, realistic, appropriate to their level of development, nonjudgmental, culturally sensitive and positive. ${ }^{56}$ Although many studies demon- 
strate success with educating adolescents about HIV infection, success in changing at risk sexual behaviour has been more limited.

The HIV antibody test became widely available in the UK in 1985 . There has been great debate about the merits of being tested for HIV infection since then. However, as the prevalence of HIV infection increases and the therapeutic options for those infected improve, the advantages of early HIV testing are becoming more apparent. Despite the advantages of knowing whether or not one is HIV antibody positive, the ethical issues regarding testing of minors are complex. Preand post-test HIV counselling must be at an appropriate level for the teenager's stage of development. The implications and limitations of both a positive and negative test should be made clear. The pros and cons of knowing HIV antibody status should be explained. Ongoing support should be available to those who seek testing particularly as an adolescent's response to testing might be even less predictable than that of an adult. Confidentiality must be assured. Whether or not an adolescent can truly give informed consent to HIV testing will vary between indi-

- viduals and depend on their intelligence, age, emotional maturity and degree of social support they have. Each case should be judged on its merits.

Homosexual adolescents In many communities there is considerable social stigma attached to homosexuality. This stigma influences the process by which teenagers become aware of their sexual orientation. Feelings of homosexuality may be dismissed or denied as may the risks of sexual experimentation. In addition, gay teenagers often face family and/or peer disapproval. This period of sexual adjustment puts gay teenagers at particular risk of STDs including HIV. This is partly because sex is often unplanned and partly because of the difficulty of forming stable relationships "in secret". In addition, as sexual novices they may be relatively uninformed about the risks of various sexual practices and easily persuaded that high risk practises are in fact safe.

Infection with hepatitis $\mathbf{B}$ is common among gay men with between $40 \%$ and $70 \%$ showing evidence of past infection and up to $10 \%$ evidence of chronic carriage. A safe and effective vaccine is now available and should be offered to all non-immune, practising homosexuals. Issues relating to HIV infection have been discussed separately in that section.

One of the most important parts of a consultation with a gay male adolescent is to assess his knowledge of safer sex practises and to ascertain how well he adheres to these. Of particular concern is his sexual behaviour while under the influence of drugs and alcohol. Referral to a counsellor or health adviser to stress the importance of safe sex may be appropriate. Adolescents who continue to take risks despite being aware of the dangers may be helped by ongoing counselling aimed at increasing their self esteem and at finding ways to avoid "risky" situa- tions. In addition they should be encouraged to attend for regular, routine STD checkups even when asymptomatic.

Sexually transmitted infections occur less commonly in lesbians than in heterosexual women; however, they do occur. There do not appear to be any STDs which occur more commonly or exclusively in lesbians and screening for STDs, if appropriate, should be as for heterosexual women. As lesbians do get cervical cancer they should be advised to have regular cervical cytology screening. ${ }^{57}$

Sexually transmitted infections and rape Rape of both men and women occurs most commonly in adolescents. In $1984,21 \%$ of female rape victims were aged 15 years or younger and $66 \%$ were under 25 years old. ${ }^{58}$ Although male rape does occur it appears to be much less common than rape in women. Again, the majority of cases occur in those who are 24 years or younger. ${ }^{58}$

Sexually transmitted infections are important in the context of rape for two reasons. The victim may acquire symptomatic or asymptomatic infections which if untreated may lead to some of the serious sequelae previously mentioned. Specimens taken for STD screening may be important forensic evidence.

Sexually transmitted infection and sexual abuse Sexually transmitted infections occurring in adolescents, particularly those who deny sexual activity may be a sign of sexual abuse. Again the infection is important both for its own sake, the psychological and psychosexual consequences and for its importance as forensic evidence.

STD prevention in the future The current increase in STDs among adolescents and the serious consequences that may follow infection must make the prevention of STDs in this age group a priority. Ideally teenagers should delay the onset of sexual activity, should always use condoms and limit the number of their sexual partners. As we have only limited knowledge about what influences teenage sexual behaviour, research directed towards this area may be valuable for future STD prevention strategies. One study has demonstrated that teenagers exposed to appropriate sex education tended to delay first intercourse, use contraception when they had intercourse and avoid pregnancy. ${ }^{59}$ Sex education is likely to reach the greatest number of teenagers if it is widely distributed via, for example schools, health care providers, television, radio, magazines, and youth groups. The information given should be accurate and non conflicting. It is likely that the key to STD prevention is to delay teenage sexual activity until the time when they have learnt sexual decision making values and skills. ${ }^{2}$

In addition to modifying sexual behaviour in order to prevent the spread of STDs, sexually active teenagers should have easy access to appropriate clinic services. They 
should be seen by clinicians with specialised training both in the management of STDs and in adolescent health care. Methods for the early detection and treatment of STDs among teenagers should be developed.

1 Premarital sexual experience among adolescent womenUnited States, 1970-1988. MMWR 1991;39:929-32.

2 Cates W. Teenagers and sexual risk taking: the best of times and the worst of times. $\mathcal{F}$ Adolesc Health Care $1991 ; 12: 84-94$

3 McAnarney ER, Hendee WR. Adolescent pregnancy and its consequences. $\mathcal{F} A M A 1989 ; 262: 74-7$.

4 Remafedi GJ. Preventing the transmission of AIDS during adolescence. f Adolesc Health Care 1988;9:139-43.

5 MacDonald NE, Wells GA, Fisher WA, et al. High-ris STD/HIV behaviour among college students. $\mathcal{F} A M A$ 1990;263:3155-9.

6 Finkl ML, Finkl DJ. Sexual and contraceptive knowledge, attitudes and behaviour of male adolescents. In Furstenberg FF, Lincoln R, Menken J, eds. Teenage sexuality, preqnancy and childbearing. Philed

7 Duncan ME, Tibaux G, Pelzer A, et al. First coitus before menarche and risk of sexually transmitted disease. menarche and risk of

8 Cohen MS, Weber RD, Mardh PA. Genitourinary mucosal defences. In: Holmes KK, Mardh PA, Sparling FP, et al, eds. Sexually Transmitted Diseases. New York: McGraw-Hill, 1990.

9 Earls F, Robins LEN, Stiffman AR, Powell J. Comprehensive health care for high risk adolescents: an evaluation study. Am J Public Health 1989;79: 999-1005.

10 Orr DP, Beiter M, Ingersoll G. Premature sexual activity as an indicator of psychosocial risk. Pediatrics 1991; 87:141-7.

11 Donovan JE, Jessor R. Structure of problem behavior in adolescence and young adulthood. $\mathcal{F}$ Consult Clin Psychol 1985;9:396-404.

12 Kraft P, Rise J, Traeen B. The HIV epidemic and changes in the use of contraception among Norwegian adolescents. AIDS 1990;4:673-8.

13 Marks A, Fisher M. Health assessment and screening during adolescence. Communicable Disease Repor Pediatric Suppl 1987;80:1(2).

14 Catchpole MA. Sexually transmitted diseases in England and Wales: 1981-1990. Communicable Disease Repor 1992;2:R1-R6.

15 Whatley J, Thin N, Reynolds B, Blackwell A. Problems of adolescents sexuality. $\mathcal{F} R$ Soc Med 1989;82:732-4.

16 Mulcalchy FM, Lacey CJN. Sexually transmitted infections in adolescent girls. Genitourin Med 1987;63: 119-21.

17 Hein K, Marks A, Cohen MI. Asymptomatic gonorrhoea. Prevalence in a population of urban adolescents. $\mathcal{F}$ Pediatr 1977;90:634-5.

18 Schafer MA, Prager V, Shalwitz J, et al. Prevalence of urethral Chlamydial trachomatis and Neisseria gonorrhoeae among asymptomatic, sexually active adolescent boys. $\mathcal{f}$ Infect Dis 1987;156:223-4.

19 Schafer MA, Beck A, Blain B, et al. Chlamydia trachomatis: important relationships to race, contraception, lowe genital tract infection and papanicolaou smears. Pediatr 1984;104:141-6.

20 Fraser JJ, Retig PT, Kaplan DW. Prevalence of cervical Chlamydial trachomatis and Neiserria gonorrhoeae in female adolescents. Pediatrics 1983;71:333--6.

21 Bell TA, Farrow JA, Stamm WE, Critchlow CW, Holmes KK. Sexually transmitted diseases in females in a juvenile detention center. Sex Transm Dis $1985 ; 12: 140-4$.

22 Fisher M, Swenson PD, Risucci D, Kaplan MH. Clinica and laboratory observations-Chlamydia trachomatis in suburban adolescents. $\mathcal{F}$ Pediatr 1987;111:617-20.

23 Hughes EG, Mowatt J, Spence JEH. Endocervica Chlamydia trachomatis infection in Canadian adolescents. Can Med Assoc F 1989;140:297-301.

24 Larsson S, Ruden AK, Bygdeman SM. Screening for Chlamydia trachomatis genital infection in young men in Stockholm. Int $\mathcal{F}$ STD AIDS 1990;1:205-6.

25 Chacko MR, Lovchik JC. Chlamydia trachomatis infection in sexually active adolescents: Prevalence and risk factors. Pediatrics 1984;73:836-40.

26 Stamm WC, Koutsky LA, Beneditti JK, Jourden L, Brunham B, Holmes KK. Chlamydia trachomatis urethral infection in men. Ann Int Med 1984;100:47-51.

27 Bell TA, Holmes KK. Age specific risks of syphilis, gonorrhoea and hospitalised pelvic inflammatory disease in sexually experienced US women. Sex Transm Dis 1984 11:291-5.

28 Westrom L. Incidence, prevalence and trends of acute pelvic inflammatory disease and its consequences in industrialised countries. Am $\mathcal{f}$ Obstet Gynecol 1980 138:880-92.
29 Spence MR, Adler J, McLellan R. Pelvic inflammatory disease in the adolescent. If Adolesc Health Care 1990 $11.304-9$.

30 Hedberg E, Anberg A. Gonococcal salpingitis: views on treatment and prognosis. Fertil Steril 1965;16:125-9.

31 Viberg L. Acute inflammatory conditions of the uterin adnexae. Acta Obstet Gynecol Scand 1964;43:5-86.

32 Golden N, Neuhoff S, Cohen H. Pelvic inflammatory disease in adolescents. F Pediatr 1989;114:138-43.

33 Litt IF, Cuskey WR. Compliance with medical regimen during adolescence. Pediatr Clin N Am 1980;27:3-15.

34 Rolfs RT, Nakashima AK. Epidemiology of primary and secondary syphilis in the United States, 1981 through 1989. $₹ A M A$ 1990;264:1432-7.

35 Alternative case finding methods in a crack related syphilis epidemic-Philadelphia. MMWR 1991;40:77-80.

36 Leads from the MMWR. Continuing increase in infectious syphilis-United States. $\mathscr{f} A M A$ 1988;259:975-6.

37 Congenital syphilis-New York City 1986-88. MMWR 1989;38:825-9.

38 Johnson RE, Nahmius AJ, Magder MPH, Lee FK, Brooks CA, Snowden CB. A seroepidemiologic survey of the prevalence of Herpes simplex virus type 2 infection in the United States. N Engl f Med 1989;321:7-12.

39 Stavraky KM, Rawls WE, Chiavetta J, Donner AP, Wanklin JM. Sexual and socioeconomic factors affectin the risk of past infections with herpes simplex virus type 2. Am ₹ Epidemiol 1983;118:109-21.

40 Koutsky LA, Ashley RL, Holmes KK, et al. The frequency of unrecognised Type 2 Herpes simplex virus infection among women. Sex Transm Dis 1990;17:90-4

41 Koutsky LA, Galloway DA, Holmes KK. Epidemiology of genital human papillomavirus infection. Epidemiological Revievs $1988 \cdot 10 \cdot 122-62$.

42 Zaninetti P, Franceschi S, Baccolo $M$, et al. Charac teristics of women under 20 with cervical intraepithelial neoplasia. Int $\mathcal{F}$ Epidemiol 1986;15:477-82.

43 Cook GA, Draper GJ. Trends in cervical cancer and carcinoma in situ in Great Britain. Br $\mathcal{f}$ Cancer 1984; 50:367-75.

44 Mitchel H, Drake M, Medley G. Prospective evaluation of risk of cervical cancer after cytological evidence of human papilloma virus infection. Lancet 1986;i:573-5.

45 Moscicki AB, Winkler B, Irwin CE, Schacter J Differences in biologic maturation, sexual behaviour, and sexually transmitted disease between adolescents with and without cervical intra-epithelial neoplasia. $f$ with and without cervical

46 Hein $\mathrm{K}$. Commmentary on adolescent acquired immunodeficiency syndrome: the next wave of the human immunodeficiency virus epidemic? $\mathcal{F}$ Pediatr 1989;114: $144-9$.

47 Burke DS, Brudage JF, Goldenbaum M, Gardener LI, Peterson $M$, Visintine $R$, Redfield $R$ and the Walte Reed Retrovirus research group. Human immunodeficiency virus infections in teenagers: seroprevalence among applicants for US military service. $\mathcal{F} A M A$ 1990;263:2074-7.

48 Wendell D, Onorato I, Allen D, et al. HIV seroprevalence among adolescents and young adults in selected clinical among adolescents and young adults in selected clinical settings, United States, 1988-90. VIth International
Conference on AIDS, San Francisco 1990 (Abstract Conferenc

49 Cowan MJ, Hellman D, Chudwin D, Wara DW, Chan RS, Ammann AJ. Maternal transmission of acquire immunodeficiency syndrome. Pediatrics 1984;73:382.

50 Novick LF, Berns D, Stricof R, et al. HIV seroprevalence in new borns in New York State. $\mathcal{f A M A} 1989$ 261:1745-50.

51 Holmberg SD, Curran JW. The epidemiology of HIV infection in industrialised countries. In: Holmes $\mathrm{KK}$ Mardh PA, Sparling FP, et al, eds. Sexually Transmitted Diseases. New York: McGraw-Hill, 1990.

52 Fischl MA, Dickinson GM, Scott GB, Klimas N, Fletcher MA, Parks W. Evaluation of heterosexual partners, chil-
dren and household contacts of adults with AIDS. dren and household con

53 Jaffe LR, Seehaus M, Wagner CF, Leadbeater B. Ana intercourse and knowledge of acquired immunodeficiency syndrome among minority-group female adolescents. F Pediatr 1988;112:1005-7.

54 Remafedi G. Adolescent homosexuality. Pediatrics 1987;79:331-7.

55 Hernandez JT, Smith FJ. Inconsistencies and misconceptions putting college students at risk of HIV infection. $\mathcal{f}$ Adolesc Health Care 1990;11:295-7.

56 Soloman MZ, Dejong W. Recent sexually transmitted disease prevention efforts and their implications for AIDS 301-16.

57 Owen WF. Medical problems of the homosexual adolescent. $\mathcal{F}$ Adolesc Health Care 1985;6:278-85.

58 Violent crime: characteristics of victims and circumstances of recorded offences 1984. Home Office Statistical Bulletin 1986;29.

59 Marsiglio W, Mott FL. The impact of sex education on sexual activity, contraceptive use and premarital pregnancy among American teenagers. Fam Plann Perspect 1986;18:151-62. 